Екушева Е.В. ${ }^{1}$, Карпова М.И. ${ }^{2}$, Осипова В.В., ${ }^{3,5}$

${ }^{\prime}$ Академия постдипломного образования ФГБУ «Федеральный научно-клинический центр специализированных видов медицинской помощи и медицинских технологий Федерального медико-биологического агентства», Москва, Россия; ${ }^{2}$ ФГБОУ ВО «Южно-Уральский государственный медицинский университет» Минздрава России, Челябинск, Россия;

${ }^{3}$ ФГАОУ ВО «Первый Московский государственный медицинский университет им. И.М. Сеченова (Сеченовский университет)» Минздрава России, Москва, Россия; ${ }^{\top}$ ГБУЗ «Научно-практический психоневрологический центр

им. З.П. Соловьева Департамента здравоохранения города Москвы», Москва, Россия;

${ }^{5} О О О$ «Университетская клиника головной боли», Москва, Россия

${ }^{1}$ 125371, Москва, Волоколамское шоссе, 91; 2454092, Челябинск, ул. Воровского, 64;

${ }^{3} 119991$, Москва, ул. Трубецкая, 8, стр. 2; ${ }^{4} 115419$, Москва, ул. Донская, 43;

${ }^{5} 121467$, Москва, ул. Молодогвардейская, 2, корп. 1

\title{
Гормональные контрацептивы
}

\section{и риск ишемического инсульта у женщин с мигренью: новый международный консенсус}

Статья посвящена безопасному применению гормональной контрацепции у женщин с мигренью - актуальной междисциплинарной проблеме, с которой ежедневно сталкиваются гинекологи, неврологи и специалисты в области диагностики и лечения головной боли. Приведены сведения о распространенности мигрени, риске ишемического инсульта и других сердечно-сосудистых нарушений у женщин с различными формами мигрени, в том числе у получающих противозачаточную и заместительную гормональную терапию. В статье приведены основные положения нового междисциплинарного консенсуса «Гормональные контрацептивы и риск ишемического инсульта у женщин с мигренью», которые рекомендуется учитывать специалистам любого профиля при назначении гормональной терапии женщинам с мигренью во избежание развития сердечно-сосудистых осложнений.

Ключевые слова: мигрень у женщин; мигрень с аурой; гормональная контрацепция; сердечно-сосудистые события; риск ишемического инсульта.

Контакты: Евгения Викторовна Екушева; ekushevaеv@таil.ru

Для ссылки: Екушева ЕВ, Карпова МИ, Осипова ВВ. Гормональные контрацептивы и риск ишемического инсульта у женщин с мигренью: новый международный консенсус. Неврология, нейропсихиатрия, психосоматика. 2019;11(3):11-15.

Hormonal contraceptives and the risk of ischemic stroke in women with migraine: a new international consensus Ekusheva E.V.', Karpova M.I. ${ }^{2}$, Osipova V.V.3,5

${ }^{1}$ Academy of Postgraduate Education «Federal Research and Clinical Center for Specialized Medical Care Types and Medical Technologies, Federal Biomedical Agency of Russia», Moscow, Russia; ${ }^{2}$ South Ural State Medical University, Ministry of Health of Russia, Chelyabinsk,

Russia; ${ }^{3}$ I.M. Sechenov First Moscow State Medical University (Sechenov University), Ministry of Health of Russia, Moscow, Russia; ${ }^{4}$ Z.P. Solovyev Research and Practical Center of Psychoneurology, Moscow Healthcare Department, Moscow, Russia; ${ }^{5} \mathrm{OOO}$ «University Clinic of Headache», Moscow, Russia

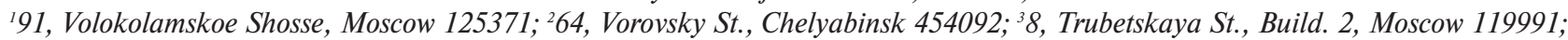
44, Donskaya St., Moscow 115419; ${ }^{5} 2$, Molodogvardeiskaya St., Build. 1, Moscow 121467

The paper is devoted to the safe use of hormonal contraception in women with migraine, an urgent interdisciplinary problem daily faced by gynecologists, neurologists, and specialists in headache diagnosis and treatment. It gives information on the prevalence of migraine, the risk of ischemic stroke and other cardiovascular disorders in women with different types of migraine, including those receiving contraception and hormone replacement therapy. The paper presents the main provisions of the new interdisciplinary consensus «Hormonal contraceptives and the risk of ischemic stroke in women who have migraine», which are recommended to be taken into account by specialists of any profile when prescribing hormonal therapy to women with migraine in order to avoid cardiovascular events.

Keywords: migraine in women; migraine with aura; hormonal contraception; cardiovascular events; risk of ischemic stroke.

Contact: Evgenia Viktorovna Ekusheva; ekushevaev@mail.ru

For reference: Ekusheva EV, Karpova MI, Osipova VV. Hormonal contraceptives and the risk of ischemic stroke in women with migraine: a new international consensus. Nevrologiya, neiropsikhiatriya, psikhosomatika = Neurology, Neuropsychiatry, Psychosomatics. 2019; $11(3): 11-15$.

DOI: $10.14412 / 2074-2711-2019-3-11-15$

По данным оценки Глобального бремени заболеваний 2016 г. (Global Burden of Disease, 2016), мигрень наряду с головной болью напряжения и лекарственно-индуцирован- ной головной болью входит в тройку самых распространенных неврологических заболеваний [1]. Согласно эпидемиологическим исследованиям, в мире частота мигрени состав- 
ляет 13-15\%, или около 1 млрд человек; в России эти цифры достигают 20,8\%, или более 29 млн человек [1-3]. В 3 раза чаще мигрень наблюдается у женщин репродуктивного возраста и наиболее распространена в возрасте 30-49 лет (24,4\%) [4-6]. В период перименопаузы у женщин мигрень также отмечается в 2 раза чаще, чем у мужчин.

\section{Мигрень и риск сердечно-сосудистых за боле в аний}

В последние два десятилетия накоплены данные, позволяющие рассматривать мигрень как один из факторов риска развития ишемического инсульта (ИИ) и других сердечно-сосудистых заболеваний. Как показал анализ историй болезни более 6,5 тыс. пациентов с мигренью, у 13,1\% из них в течение жизни обнаруживались сопутствующие сердечно-сосудистые заболевания [7], а вероятность состоявшихся сосудистых эпизодов увеличивалась по мере нарастания частоты приступов головной боли в месяц [8]. Важно отметить, что в женской популяции в большей степени, чем в мужской, мигрень с аурой является фактором риска цереброваскулярных и кардиоваскулярных заболеваний, в частности инфаркта миокарда, нестабильной стенокардии, транзиторных ишемических атак (ТИА) и ИИ, причем вероятность возникновения ИИ и ТИА у женщин в 3 раза выше, чем у мужчин [8, 9].

При анализе причин развития ИИ у молодых женщин (до 35 лет) установлено, что единственным значимым фактором риска состоявшейся сосудистой катастрофы было наличие мигрени с аурой ( $\mathrm{p}=0,003)$, в то же время увеличения риска развития геморрагического инсульта не наблюдалось [10, 11]. Показано, что возникновение мигренозной ауры на протяжении 1 года ассоциируется с повышенным риском ИИ: при появлении ауры реже 1 раза в месяц этот риск был увеличен в 2 раза, чаще 1 раза в месяц - в 4 раза [12]. Отмечено также, что риск развития цереброваскулярных и кардиоваскулярных заболеваний у пациентов с мигренью существенно выше при наличии других факторов сосудистого риска, наибольшее значение среди которых имеют женский пол, курение, артериальная гипертензия, ожирение и применение эстрогенсодержащих гормональных препаратов [6, 13].

\section{Мигрень игормональная терапия}

Известно, что циклические колебания половых гормонов в разные периоды жизни могут оказывать значительное влияние на течение мигрени у женщин. Так, частота, интенсивность, длительность, характер и особенности приступов головной боли могут изменяться в разные фазы менструального цикла, во время беременности, лактации, перименопаузы, приема гормональных контрацептивов и заместительной гормональной терапии $[14,15]$.

Противозачаточная и заместительная гормональная терапия широко применяется во всем мире у женщин в репродуктивном и перименопаузальном периодах. За последние несколько десятилетий произошла значительная эволюция средств для гормональной контрацепции, в том числе комбинированных оральных контрацептивов (КОК), которые в зависимости от поступающей в организм суточной дозы этинилэстрадиола делятся на высокодозированные (40 мкг/сут), низкодозированные (30-35 мкг/сут) и микродозированные (15-20 мкг/сут) [16]. Разработка новых форм, режимов и путей введения гормональных препаратов способствовала существенному уменьшению нежелательных эффектов и неблагоприятных патологических состояний.

Известно, что в постменопаузальном периоде на фоне естественного снижения уровня эстрогенов риск инсульта у женщин существенно увеличивается, причем еще более высокий риск отмечается при приеме КОК [9]. Как видно из табл. 1, риск развития ИИ у женщин 20-44 лет, принимающих КОК и имеющих мигрень без ауры, составляет 25,4 на 100 тыс., а у имеющих мигрень с аурой - 36,9 на 100 тыс. [17].

Абсолютный риск ИИ у женщин 20-44 лет в зависимости от наличия мигрени и приема гормональных контрацептивов [17]

Отсутствие Мигрень Мигрень

\begin{tabular}{|llll|}
\hline $\begin{array}{l}\text { Прием гормональных } \\
\text { контрацептивов }\end{array}$ & $\begin{array}{l}\text { Отсутствие } \\
\text { мигрени }\end{array}$ & $\begin{array}{l}\text { Мигрень } \\
\text { без ауры }\end{array}$ & $\begin{array}{l}\text { Мигрень } \\
\text { с аурой }\end{array}$ \\
\hline Нет & $2,5 / 100000$ & $4,0 / 100000$ & $5,9 / 100000$ \\
\hline Да & $6,3 / 100000$ & $25,4 / 100000$ & $36,9 / 100000$ \\
\hline
\end{tabular}

Важно заметить, что курение в 7 раз повышает вероятность цереброваскулярных эпизодов у женщин с мигренью с аурой, использующих КОК [17]. При приеме гормональных контрацептивов выявлен повышенный риск тромбофилических осложнений $[6,10,13]$, в ряде исследований обнаружена ассоциация состоявшихся кардиальных и цереброваскулярных эпизодов у женщин с мигренью, принимавших KOK [13, 17, 18].

\section{Гормональная терапия и риск}

\section{с ердечн о - сосудистых заболеваний}

Хотя доказана взаимосвязь гормональной терапии и риска возникновения сердечно-сосудистых событий, в последнее время ведется активная дискуссия о зависимости степени этого риска от дозы этинилэстрадиола в препаратах. Результаты крупных эпидемиологических исследований последних лет убедительно показывают, что угроза сердечнососудистых осложнений возникает лишь при применении КОК, содержащих большие дозы этинилэстрадиола, в то время как современные низкодозированные и микродозированные КОК не оказывают клинически значимого влияния на различные параметры гомеостаза, крайне редко ассоциированы с развитием артериального тромбоза и являются безопасным методом предохранения от нежелательной беременности у здоровых некурящих женщин моложе 40 лет $[16,19]$. По данным метаанализа исследований, риск ИИ у женщин значительно уменьшался при использовании КОК, содержащих низкие дозы эстрогена ( $\leqslant 50$ мкг) [20]. Показано, что риск тромбозов также может коррелировать не только с дозой эстрогенов, но и с продолжительностью гормональной терапии [21]. При этом длительное применение микродозированных КОК, содержащих $\leqslant 15$ мкг этинилэстрадиола, не только не увеличивает риск цереброваскулярных эпизодов [21], но и способствует профилактике различных патологических состояний (например, уменьшает частоту и выраженность приступов менструальной мигрени) [22, 23] и может оказывать лечебно-профилактический эффект в от- 
Таблица 2. Скрининговый диагностическийопросникдля идентификаиии мигрени (ID-Migraine)

Ответьте на следующие три вопроса

Сопровождалась ли у вас головная боль за последние 3 мес следующими симптомами:

1. Тошнотой или рвотой? Да Нет

2. Непереносимостью света и звуков? Да Нет

3. Негативным влиянием на работоспособность, учебу или повседневные дела как минимум в течение 1 дня?

Да Нет

Таблица 3. Шкала оценки зрительной ауры [27]

\begin{tabular}{|lc|}
\hline Зрительные и феномены & Оценка риска \\
\hline Продолжительность 5-60 мин & 3 \\
\hline Постепенное развитие в течение 5 мин & 2 \\
\hline Скотома & 2 \\
\hline Зигзагообразные линии & 2 \\
\hline Односторонняя гемианопсия & 1 \\
\hline Установлен диагноз мигрени с аурой & $\geqslant 5$ \\
\hline
\end{tabular}

ношении некоторых гинекологических заболеваний (злокачественные процессы в яичниках и матке) [24, 25].

В то же время большая часть систематизированных обзоров и метаанализов, демонстрирующих взаимосвязь цереброваскулярных и кардиоваскулярных событий у женщин с мигренью с аурой, принимающих КОК, базируется на данных 20-30-летней давности, при этом подчеркивается, что подавляющее число этих эпизодов отмечалось при применении высокодозированных гормональных средств (с содержанием этинилэстрадиола $\geqslant 50$ мкг) [21]. Поскольку именно в женской популяции мигрень является значимым фактором риска цереброваскулярных и кардиоваскулярных заболеваний $[8,9]$, вероятность возникновения которых повышается при применении гормональной контрацепции, возможность, целесообразность и безопасность использования этих лекарственных средств являются чрезвычайно актуальной проблемой.

\section{Современные рекомендации по назначению гормональных контрацептивов женщинам с мигренью}

Противоречивость взглядов на использование гормональной контрацепции у пациенток с мигренью и накопленные в последние годы данные о соотношении пользы и риска такой терапии в отношении ИИ послужили основой для междисциплинарного обсуждения этой актуальной проблемы независимыми экспертами в области головной боли и гормональной контрацепции. Результатом этого обсуждения стал междисциплинарный консенсус «Гормональные контрацептивы и риск ишемического инсульта у женщин c мигренью», разработанный специалистами Европейской федерации головной боли и Европейского общества контрацепции и репродуктивного здоровья [17]. Этот документ, опубликованный в 2017 г., содержит 13 положений, уточняющих характер и последовательность шагов при выборе адекватного противозачаточного средства у женщин с миг- ренью. Эти положения были сформулированы на основании большого числа исследований с учетом уровня убедительности рекомендации (УУР) и уровня достоверности доказательств (УДД); для клиницистов (как неврологов, так и гинекологов) представленная информация имеет рекомендательный характер.

Согласно консенсусу, при выборе варианта гормональной контрацепции в первую очередь следует выяснить у женщины, не беспокоят ли ее периодически повторяющиеся головные боли и, если да, то установить их природу. В повседневной практике для целенаправленной диагностики мигрени удобно использовать скрининговый опросник идентификации мигрени (табл. 2). При получении положительных ответов хотя бы на два вопроса этого теста можно с вероятностью в $93 \%$ предполагать наличие мигрени [26].

Поскольку наличие мигрени с аурой сопряжено с самым большим риском сердечно-сосудистых событий, следующим необходимым шагом является уточнение наличия ауры, предшествующей фазе головной боли. С этой целью может быть использована краткая шкала оценки зрительной ауры (табл. 3).

После установления диагноза мигрени и определения ее формы (мигрень с аурой или без ауры) необходимо принять решение о выборе препарата для контрацепции (табл. 4). Как уже упоминалось выше, определяющим фактором при этом является содержание этинилэстрадиола:

- наибольший риск ИИ отмечается при приеме КОК, содержащих >35 мкг этинилэстрадиола;

- средний риск - при использовании комбинированных гормональных контрацептивов, содержащих <35 мкг этого гормона;

- отсутствие риска при приеме прогестагенсодержащих лекарственных средств [17].

Последним важным этапом при выборе контрацептивного средства является анализ дополнительных факторов риска сердечно-сосудистых заболеваний, при наличии которых рекомендуются негормональные (презервативы, медьсодержащая внутриматочная спираль, диафрагма и др.) или прогестагенсодержащие контрацептивы [17].

Большое значение имеет оценка состояния женщины на фоне приема гормональных контрацептивов. В связи с этим женщины без мигрени, применяющие гормональную контрацепцию, должны сообщать лечащему врачу о появлении любой головной боли, а женщины с мигренью - о любых изменениях в течении цефалгии (например, об учащении приступов головной боли, первом появлении ауры, изменении характера головной боли и/или симптомов ауры). При наличии этих нарушений женщина должна немедленно прекратить прием гормональных препаратов и обратиться к лечащему врачу, который перенаправит ее к неврологу. Если женщина курит, следует разъяснить ей необходимость отказа от курения до начала приема гормональных контрацептивов. Важно обратить внимание пациентки на необходимость коррекции и других факторов риска цереброваскулярной и кардиоваскулярной патологии (в первую очередь снижение массы тела, контроль артериального давления и уровня сахара в крови). 
Таблица 4. Основные положенияконсенсуса "Гормональные контрацептивы ириск ишемического инсульта у женщин смигренью» (по [17] с изменениями)

Основные положения

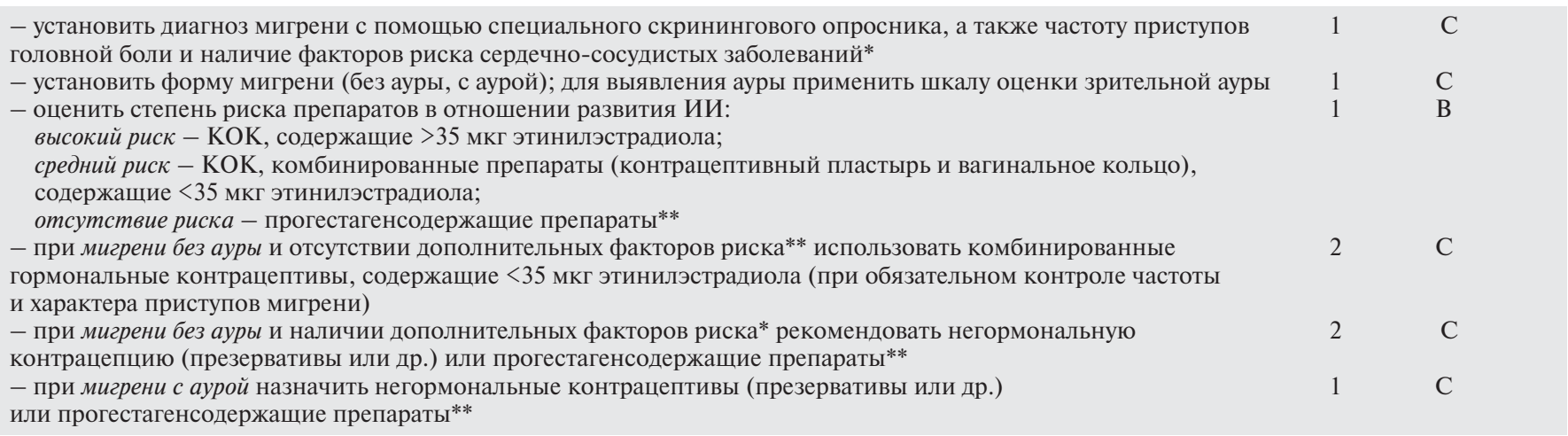

Женщинам с мигренью без ауры и наличием дополнительных факторов риска*, уже использующим комбинированные этинилэстрадиолсодержащие контрацептивы:

рекомендовано отменить эти препараты и перейти на прием негормональных контрацептивов или прогестагенсодержащих препаратов**

Примечание. Уровень $1 \boldsymbol{B}$ (доказательства основаны на результатах рандомизированных контролируемых исследований с определенными ограничениями, в том числе касающихся методологии) - рекомендовано всем пациенткам, преимущества явно перевешивают риски и бремя заболевания; уровень 1 C (доказательства основаны на результатах наблюдательных исследований или описаниях серии случаев) - рекомендовано при отсутствии доказательств более высокого уровня, преимущества явно перевешивают риски и бремя заболевания;

уровень $2 C$ (доказательства основаны на результатах наблюдательных исследований или описаниях серии случаев) - невысокий уровень доказательности, не определено соотношение пользы, риска и бремени. ${ }^{*}-$ курение, артериальная гипертензия, ожирение, наличие в анамнезе сердечно-сосудистых заболеваний, тромбоза глубоких вен или тромбоэмболии легочной артерии; ** - пероральная таблетка, субдермальный имплантат, депо-инъекция и левоноргестрел-высвобождающая внутриматочная система.

\section{З а к лю че ни и}

Таким образом, наличие у женщины мигрени определяет необходимость выбора адекватного и безопасного метода контрацепции в зависимости от формы заболевания (мигрень без ауры или с аурой), частоты приступов головной боли и наличия дополнительных факторов риска цереброваскулярной и кардиоваскулярной патологии. Для выявления мигрени, в том числе мигрени с аурой, разработаны удобные и простые анкеты, которые могут использоваться клиницистами, назначающими гормональную терапию. Наблюдение за клиническими особенностями и динамикой головной боли на протяжении курса гормональной терапии является необходимым условием ее безопасности и своевременного предотвращения возможных сердечно-сосудистых осложнений.

Для подбора безопасных средств контрацепции у женщин с мигренью большую роль играют взаимодействие невролога, гинеколога или врача другой специальности, назначающего гормональные контрацептивы или заместительную гормональную терапию, а также сотрудничество с пациенткой, что позволяет избежать нежелательных эффектов, осложнений и контролировать проводимое лечение.

\section{Л И Т Е Р A Т V}

1. Global, regional, and national incidence, prevalence, and years lived with disability for 328 diseases and injuries for 195 countries, 1990-2016: a systematic analysis for the Global Burden of Disease Study 2016. Lancet Neurol. 2017;390:1211-1859. doi:10.1016/S0140-6736 (17)32154-2

2. Tietjen GE, Collins SA. Hypercoagulability and migraine. Headache. 2018;58(1):173-183. http://dx.doi.org/10.1111/head.13044 3. Ayzenberg I, Katsarava Z, Sborowski A, et al. The prevalence of primary headache disorders in Russia: A countrywide survey. Cephalalgia. 2012 Apr;32(5):373-81. doi: 10.1177/ 0333102412438977. Epub 2012 Mar 6. 4. Осипова ВВ, Табеева ГР. Первичные головные боли: диагностика, клиника, тера- пия: практическое руководство. Москва: МИА; 2014. 336 с. [Osipova VV, Tabeeva GR. Pervichnye golovnye boli: diagnostika, klinika, terapiya: Prakticheskoe rukovodstvo [Primary headaches: diagnosis, clinic, therapy: a practical guide]. Moscow: MIA; 2014. 336 p.] 5. Екушева ЕВ. Оптимальные подходы к купированию приступа мигрени: прошлое, настоящее и будущее. Русский медицинский журнал. 2012;(10):522-8. [Ekusheva EV. Optimal approaches to arresting a migraine attack: past, present and future. Russkii meditsinskii zhurnal. 2012;(10):522-8 (In Russ.)]. 6. Finocchi C, Strada L. Sex-related differences in migraine. Neurol Sci. 2014 May;35 Suppl 1:207-13. doi: 10.1007/s10072-014-1772-y. 7. Buse DC, Reed ML, Fanning KM, et al.
Cardiovascular events, conditions, and procedures among people with episodic migraine in the US population: results from the American Migraine Prevalence and Prevention (AMPP) study. Headache. 2017 Jan;57(1):31-44. doi: 10.1111/head.12962. Epub 2016 Nov 12. 8. Peng KP, Chen YT, Fuh JL, et al. Migraine and incidence of ischemic stroke: A nationwide population-based study. Cephalalgia. 2017 Apr; 37(4):327-335. doi: 10.1177/0333102416642602. Epub 2016 Apr 26.

9. Sacco S, Ricci S, Carolei A. Migraine and vascular diseases: a review of the evidence and potential implications for management. Cephalalgia. 2012 Jul;32(10):785-95. doi: $10.1177 / 0333102412451361$.

Epub 2012 Jun 18. 
10. Carolei A, Marini C, De Matteis G. History of migraine and risk of cerebral ischaemia in young adults. The Italian national research council study group on stroke in the young. Lancet. 1996 Jun 1;347(9014):1503-6.

11. Nicodemo M, Cevoli S, Giannini G, Cortelli P. Comorbidity in perimenstrual migraine. Curr Pain Headache Rep. 2012 Oct;16(5):477-83. doi: 10.1007/s11916-0120281-z.

12. Todd C, Lagman-Bartolome AM, Lay C. Women and migraine: the role of hormones. Curr Neurol Neurosci Rep. 2018 May 31;18(7): 42. doi: 10.1007/s11910-018-0845-3.

13. Sheikh HU, Pavlovic J, Loder E, Burch R Risk of stroke associated with use of estrogen containing contraceptives in women with migraine: a systematic review. Headache. 2018 Jan;58(1):5-21. doi: 10.1111/head.13229. Epub 2017 Nov 15.

14. Vetvik KG, Benth JS, MacGregor EA, et al. Menstrual versus non-menstrual attacks of migraine without aura in women with and without menstrual migraine. Cephalalgia. 2015 Dec; 35(14):1261-8. doi: 10.1177/0333102415575723. Epub 2015 Mar 9.

15. Elliott D, Li X, Zhu P, Gaitour E.

Headache in pregnancy. In: Minagar A, editor. Neurological Disorders and Pregnancy. London: Elsevier; 2011. P. 19-37.

16. Medical eligibility criteria for contraceptive use, 5th ed. Geneva: WHO; 2015. 276 p.
17. Sacco S, Merki-Feld GS, Egidius KL, et al. Hormonal contraceptives and risk of ischemic stroke in women with migraine: a consensus statement from the European Headache Federation (EHF) and the European Society of Contraception and Reproductive Health (ESC). $J$ Headache Pain. 2017 Oct 30;18(1):108. doi: 10.1186/s10194-017-0815-1.

18. Abanoz Y, Abanoz YG, Gü ndü z A, et al. Migraine as a risk factor for young patients with ischemic stroke: a case-control study. NNeurol Sci. 2017 Apr;38(4):611-617. doi: 10.1007/s10072-017-2810-3.

Epub 2017 Jan 12.

19. The Oral Contraceptive and Hemostasis Study Group. The effects of seven monophasic oral contraceptive regimens on hemostatic variables: conclusions from a large randomized multicenter study. Contraception. 2003 Mar; 67(3):173-85.

20. Gillum LA, Mamidipudi SK, Johnston SC. Ischemic stroke risk with oral contraceptives: a meta-analysis. JAMA. $2000 \mathrm{Jul}$ 5;284(1):72-8.

21. Calhoun A, Ford S, Pruitt A. The impact of the extended-cycle vaginal ring contraception on migraine aura: a retrospective case series. Headache. 2012 Sep;52(8):1246-53. doi: 10.1111/j.1526-4610.2012.02211.x. Epub 2012 Jul 12.

22. Sulak P, Willis S, Kuehl T, et al. Headaches and oral contraceptives: impact of eliminating the standard 7-day placebo interval. Headache.
2007 Jan;47(1):27-37. doi: 10.1111/j.1526-4610. 2007.00650.x

23. Nappi RE, Kaunitz AM, Britzer J.

Extended regimen combined oral contraception: a review of evolving concepts and acceptance by woman and clinicians. Eur J Contracept Reprod Health Care. 2016;21(2):106-15. doi: 10.3109/13625187.2015.1107894.

Epub 2015 Nov 17.

24. Collaborative group. Endometrial cancer and oral contraceptives: an individual participant meta-analysis of 27276 women with endometrial cancer from 36 epidemiological studies. Lancet Oncol. $2015 \mathrm{Sep}$;16(9): 1061-1070. doi: 10.1016/S1470-2045(15)00212-0. Epub 2015 Aug 4.

25. Collaborative group. Ovarian cancer and oral contraceptives: collaborative reanalysis of data from 45 epidemiological studies including 23257 women with ovarian cancer and 87303 controls. Lancet. 2008 Jan 26;371(9609):303-14. doi: 10.1016/S0140-6736(08)60167-1. 26. Lipton RB, Dodick D, Sadovsky R, et al. A self-administered screener for migraine in primary care: The ID Migraine validation study. Neurology. 2003 Aug 12;61(3):375-82. doi: 10.1212/01.WNL.0000078940.53438.83

27. Eriksen MK, Thomsen LL, Olesen J. The visual aura rating scala (VARS) for migraine aura diagnosis. Cephalalgia. 2005 Oct; 25(10):801-10. doi: 10.1111/j.1468-2982.2005. 00955.x

\section{Поступила 8.07.2019}

Исследование не имело спонсорской поддержки. Авторы несут полную ответственность за предоставление окончательной версии рукописи в печать. Все авторы принимали участие в разработке концепции статьи и написании рукописи. Окончательная версия рукописи была одобрена всеми авторами. 